\title{
Désert Oriental (2020)
}

Stathmoi et metalla. Exploiter et traverser le désert Oriental à l'époque ptolémaïque

Thomas Faucher, Bérangère Redon, Adam Bülow-Jacobsen, Maël Crepy, Hélène Cuvigny, Jennifer Gates-Foster, Isabelle Goncalves, Mariola Hepa, Damien Laisney, Joachim Le Bomin, Julie Marchand, Alexandre Rabot, Markos Vaxevanopoulos et Noémi Villars

\section{(2) OpenEdition} Journals

Édition électronique

URL : https://journals.openedition.org/baefe/2714

DOI : $10.4000 /$ baefe. 2714

ISSN : 2732-687X

Éditeur

ResEFE

Référence électronique

Thomas Faucher, Bérangère Redon, Adam Bülow-Jacobsen, Maël Crepy, Hélène Cuvigny, Jennifer Gates-Foster, Isabelle Goncalves, Mariola Hepa, Damien Laisney, Joachim Le Bomin, Julie Marchand, Alexandre Rabot, Markos Vaxevanopoulos et Noémi Villars, « Désert Oriental (2020) » [notice archéologique], Bulletin archéologique des Écoles françaises à l'étranger [En ligne], Égypte, mis en ligne le 30 mai 2021, consulté le 25 juillet 2021. URL : http://journals.openedition.org/baefe/2714 ; DOI : https://doi.org/10.4000/baefe.2714

Ce document a été généré automatiquement le 25 juillet 2021.

Le Bulletin archéologique des Écoles françaises à l'étranger est mise à disposition selon les termes de la Licence Creative Commons Attribution - Pas d'Utilisation Commerciale - Pas de Modification 4.0 International. 


\section{Désert Oriental (2020)}

Stathmoi et metalla. Exploiter et traverser le désert Oriental à l'époque ptolémaïque

Thomas Faucher, Bérangère Redon, Adam Bülow-Jacobsen, Maël Crepy, Hélène Cuvigny, Jennifer Gates-Foster, Isabelle Goncalves, Mariola Hepa, Damien Laisney, Joachim Le Bomin, Julie Marchand, Alexandre Rabot, Markos Vaxevanopoulos et Noémi Villars

\section{NOTE DE L'AUTEUR}

Année de la campagne : 2020 ( 6 janvier - 2 février)

Autorité nationale présente : Le ministère du Tourisme et des Antiquités (MoTA) était représenté par Osama Mahdey Ahmed Mouhamed et Abdel Aleem Mouhamed Tentawy Mahmoud

Numéro et intitulé de l'opération de terrain : 17152 Désert Oriental Composition de l'équipe de terrain : L'équipe était composée de Thomas Faucher (archéologue, numismate, IRAMAT-CRP2A, Bordeaux), Bérangère Redon (archéologue, CNRS-HiSoMA, Lyon), Adam Bülow-Jacobsen (papyrologue, photographe), Charlène Bouchaud (archéobotaniste, CNRS-MNHN), Dominique Cardon (spécialiste des textiles, CNRS-Ciham), Marie-Pierre Chaufray (papyrologue, CNRS-Ausonius, Bordeaux), Maël Crepy (géographe, UMR 5189 HiSoMA, UMR 5133 Archéorient), Hélène Cuvigny (papyrologue, CNRS-IRHT, Paris), Jennifer Gates-Foster (céramologue, University of North Carolina at Chapel Hill), Isabelle Goncalves (archéologue, doctorante, université de Lyon), Mariola Hepa (archéologue, Swiss Institute for Architectural and Archaeological Research on Ancient Egypt), Damien Laisney (topographe, FR 3747 Maison de l'Orient et de la Méditerranée), Martine Leguilloux (archéozoologue, Centre archéologique du Var), Joachim Le Bomin (archéologue, UMR 5189 HiSoMA), Louis Manière (SIG, UMR 5189 HiSoMA), Julie Marchand (céramologue, UMR 5189 HiSoMA), Gaël Pollin (photographe, Ifao), Alexandre Rabot (spécialiste du SIG, HiSoMA, université Lumière Lyon 2), Markos Vaxevanopoulos (géologue, ENS Lyon), Noémi Villars (Data Manager, CNRS-HiSoMA). 
Partenariats institutionnels : La mission bénéficie du soutien du CNRS (UMR 5189

Histoire et Sources des Mondes Antiques HiSoMA et UMR 5060 Institut de Recherche sur les ArchéoMATériaux IRAMA), de l'université Lumière Lyon 2 et de University of North Carolina at Chapel Hill.

Organismes financeurs :

- Ministère de l'Europe et des Affaires étrangères (MEAE)

- ERC project Desert Networks (grant agreement No 759078)

Données scientifiques produites :

https://desorient.hypotheses.org/

\section{Problématique et bilan des principaux résultats}

1 La mission archéologique française du désert Oriental a exploré entre 2013 et 2016 le district minier de Samut. Elle a fouillé la mine d'or de Samut Nord et le fort de Bi'r Samut, tous deux datés de l'époque ptolémaïque, et localisés sur l'ancienne route menant d'Edfou au port de Bérénice. Lors des campagnes 2017 et 2018, nous avons fouillé un deuxième fortin ptolémaïque, localisé sur la même route, pour compléter les données cruciales mais isolées tirées de l'exploration du fort de Bi'r Samut. Notre choix s'était porté sur le fortin d'Abbad, situé à $22 \mathrm{~km}$ d'Edfou, à la fois pour sa localisation, sa durée d'occupation mais aussi à cause des dégradations qui avaient eu lieu sur le site peu avant nos travaux.

2 Suite à une année consacrée à l'étude du matériel en 2019, nous avons modifié notre implantation géographique en déplaçant la mission à $200 \mathrm{~km}$ au nord. Des prospections avaient en effet permis de mettre en lumière le potentiel unique du site de Ghozza, situé au nord de la route de Myos Hormos. Composé d'un village de mineurs d'époque ptolémaïque et d'un fortin du Haut Empire romain, l'ensemble se situe au cœur d'un très important district minier caractérisé par de nombreux travaux sur filon, aussi bien aériens qu'en galeries. Profitant de l'implantation de la mission dans cette zone du désert, nous avons entrepris parallèlement la fouille d'un fortin romain situé à $8 \mathrm{~km}$ au nord de Ghozza : Deir el-Atrash. Sa fondation apparemment plus tardive que le fortin romain de Ghozza permettait de compléter nos données en bénéficiant de documents plus tardifs, renseignant ici l'implantation dans cette partie du désert aux époques ptolémaïque et romaine.

\section{Ghozza}

\subsection{Le fort romain}

Isabelle Goncalves, Bérangère Redon

Le fort romain de Berkou (nom antique du site tel qu'attesté dans les ostraca mis au jour durant les fouilles) se situe sur la route de la mer Rouge, et en direction des carrières du Porphyritès et du Mons Claudianus. 
Fig. 1. Vue d'ensemble du site de Ghozza en cours de fouille (G. Pollin).

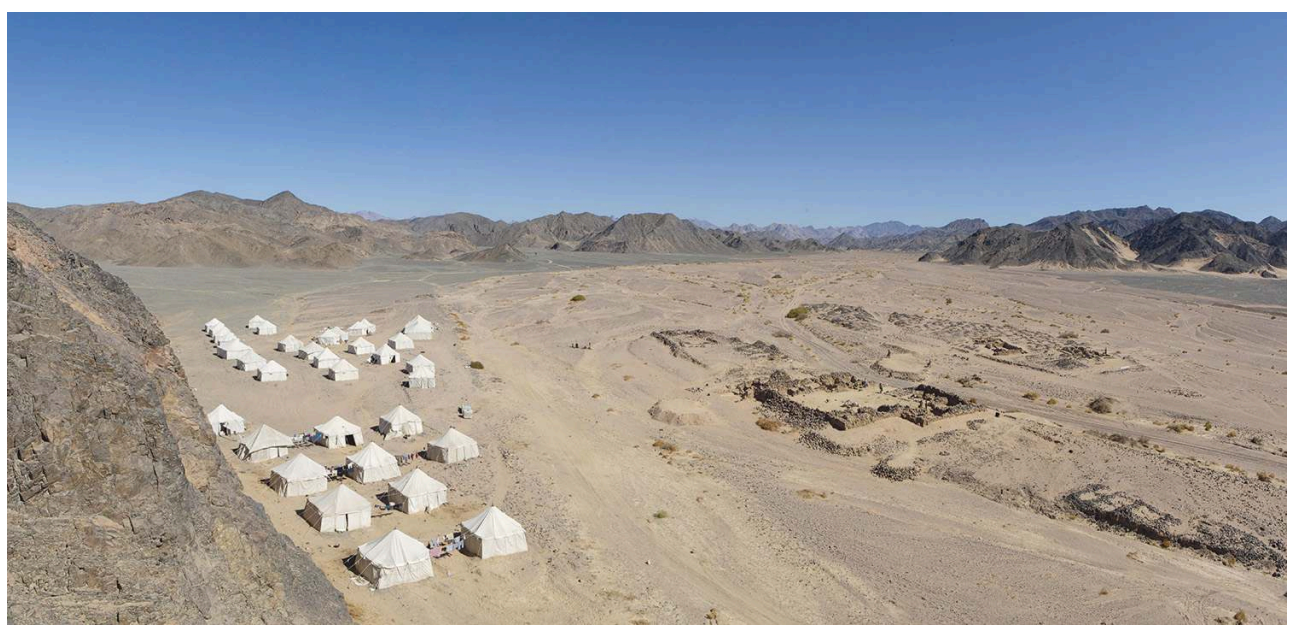

(C) MAFDO, 17152_2020_NDMPF_001

Il a été presque entièrement fouillé en 2020 ; sa taille, de moins de $1000 \mathrm{~m}^{2}(29 \mathrm{~m}$ de long et $25 \mathrm{~m}$ de large) est modeste en comparaison de celle des fortins romains construits à la même période dans le désert Oriental.

5 Le fortin est bâti autour d'une cour centrale, sur laquelle s'ouvrent au moins quinze pièces, construites contre le mur d'enceinte. La porte d'entrée a été vraisemblablement emportée par le ouadi ; elle devait se situer au sud-est. Une poterne a été bâtie dans l'angle nord; elle ouvrait sur quelques marches qui donnaient un accès direct à une citerne extérieure. Devant les pièces, sont parfois aménagées des installations légères organisées autour de petits silos ou de fours. Quatorze pièces ont été fouillées lors de la campagne 2020 ; seule la cour n'a pas fait l'objet de sondage, mise à part dans sa partie nord-ouest. Même si l'absence de dégagement d'ampleur du centre de la cour ne permet pas d'en être définitivement sûr, il semble que le fort n'a pas abrité de puits central comme cela est habituel dans les fortins romains du désert Oriental. Une citerne, profonde de 2,30 m, située à l'extérieur, au nord-est, est alimentée en eau par un puits extérieur, via une longue canalisation. Ce puits a été emporté par le ouadi et nous n'en connaissons pas les caractéristiques. Toujours à l'extérieur, contre le mur d'enceinte à l'est, un abreuvoir d'une dizaine de mètres de long est alimenté par un système de shaduf à partir de la citerne.

6 La terrasse alluviale sur laquelle repose le fort est vraisemblablement déjà occupée à l'époque ptolémaïque (voir la description du village de mineurs) même si l'emprise du, ou des, bâtiments préexistants n'est pas connue. Quoi qu'il en soit, du matériel épars mis au jour dans la plupart des couches situées sous le fort et qui ont été nivelées durant l'époque romaine permet d'en être assuré. Lors de la construction du fort romain, les constructeurs ont massivement utilisé des pierres de remploi, et notamment un grand nombre de meules à broyer le minerai, pour édifier le mur d'enceinte et les murs internes. On retrouve également ces meules dans plusieurs endroits du fort, sous la forme de dallage. Dans ce cas, il est probable qu'il s'agisse de sols édifiés à l'époque ptolémaïque et remployés, le cas échéant, dans ou aux abords du fort. Le plan du fortin, qui n'est pas strictement rectangulaire, s'appuie d'ailleurs sur des constructions antérieures, ce qui expliquerait la protubérance de l'aile ouest. 
7 La fonction des pièces reste délicate à définir puisqu'elles ont visiblement été régulièrement nettoyées (le dépotoir qui devait se situer à l'extérieur du fortin a été emporté par les crues successives). Au moins à la fin de son occupation, il est vraisemblable qu'une partie seulement des pièces étaient habitées puisque les déchets produits par les habitants du fort n'étaient plus jetés à l'extérieur mais dans certaines pièces, situées dans l'angle sud-ouest.

\subsection{Le village de mineurs}

Au sud du fort, sur une grande terrasse de $180 \mathrm{~m}$ de long et de $50 \mathrm{~m}$ de large, divisée en deux par un petit bras du ouadi, s'étale un village de mineurs. Il se compose d'un grand nombre de constructions en pierre sèche, organisées plus ou moins dans une orientation nord-sud. De ce vaste ensemble, seules deux zones ont été fouillées en 2020 : un complexe thermal, le plus grand bâtiment de l'îlot et ses environs immédiats.

\subsubsection{Les bains}

B. Redon

9 À l'extrémité sud-ouest du village se tient un édifice balnéaire dont la présence à Ghozza est révélatrice de la variété et de la complexité de la population qui a pu y habiter durant l'époque ptolémaïque.

Fig. 2. Complexe balnéaire de Ghozza (B. Redon).

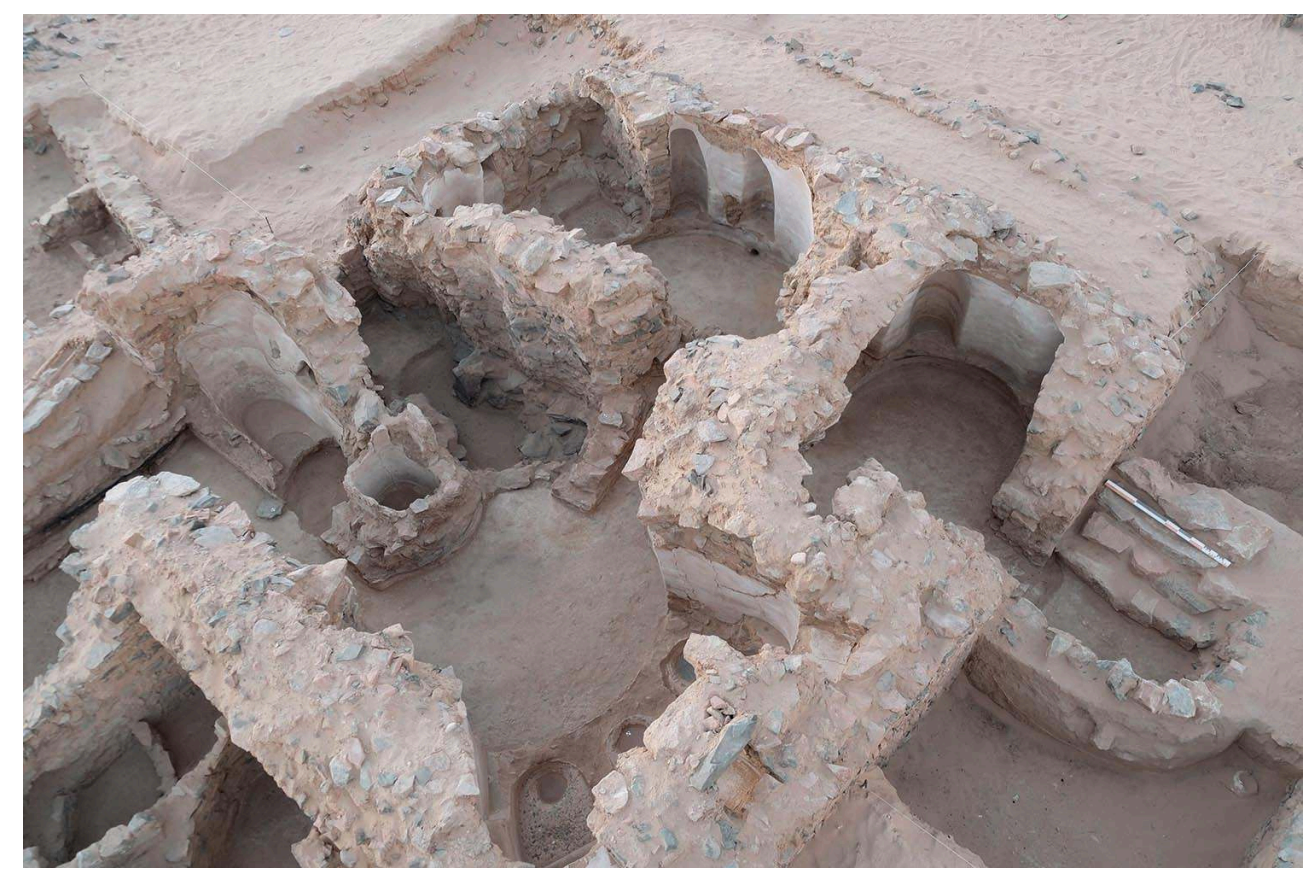

(c) MAFDO, 17152_2020_NDMPF_002

10 Il comprend trois salles balnéaires, un vestiaire, ainsi que plusieurs pièces annexes au sud et à l'ouest du bain. Une salle de service à l'air libre était située à l'ouest et donnait accès au foyer des bains situés au milieu de l'édifice.

11 Le bain de Ghozza se caractérise par sa surface limitée (environ $45 \mathrm{~m}^{2}$ ), la présence des deux équipements balnéaires classiques des bains grecs, à savoir des cuves plates (pour 
le bain d'hygiène) et deux baignoires d'immersion (pour le bain de détente), réparties dans deux pièces ouvertes aux baigneurs. Il est semblable en cela à une douzaine de «petits bains collectifs grecs » dont l'étude de synthèse a récemment paru ${ }^{1}$. Il présente néanmoins un élément jusqu'ici jamais attesté, mais largement illustré dans les papyrus : un vestiaire à l'entrée des bains. Les baigneurs y acquittaient sans doute le prix de l'entrée auprès du tenancier, et y déposaient leurs affaires.

Les murs des bains sont construits en pierres locales liées à la terre. Ils sont assez peu épais, entre 55 et $65 \mathrm{~cm}$ d'épaisseur. L'édifice était surmonté de voûtes et coupoles, dont on a retrouvé les départs dans certaines des pièces du bain. Comme les briques crues des voûtes nubiennes, les dalles étaient placées sur le champ. Murs et voûtes du bain étaient enduits de mortier de chaux de couleur blanche. Le vestiaire a fait l'objet d'un traitement un peu particulier sous la forme d'un bourrelet/ressaut du mortier, au bas de ses murs. On ne retrouve nulle part ailleurs de décor dans le bain, dont les murs sont simplement enduits de mortier de chaux, de couleur blanche à beige, sauf un dessin assez sommaire, fait au noir de charbon, laissé sur le bas du mur des vestiaires, à droite de l'entrée des bains. Il s'agit d'un personnage debout et de face, qui tient dans sa main un objet que l'on peut identifier à un outil (pic ?) ou une arme ( $\operatorname{arc}$ ?).

Les sols du bain, des cuves plates et de la baignoire sont revêtus d'une couche de surface faite de mortier, qui a été répandu sur le sol. Cette couche de surface est le plus souvent très fine, par opposition aux couches de soubassement. Ces sols de mortier sont solidifiés par l'ajout d'éclats de pierre et de galets, avec des traitements différents pour les sols de circulation et les sols des cuves plates. Les cuves présentent une autre originalité dans leur construction: le fond de leur cupule est renforcé par la présence de meules allantes dont la résistance présentait en effet de gros avantages sur la pierre locale.

Le système de chauffage du bain prend place au cœur même de l'édifice, comme il est de coutume dans les bains grecs d'Égypte. Le four est formé d'une bouche, ménagée entre deux maçonneries de pierre, dont une meule dormante placée sur le chant. Il permettait de chauffer l'eau utilisée par les baigneurs dans les cuves plates puis dans les baignoires. L'élément le plus évident du système d'approvisionnement en eau est un bassin situé à l'entrée d'une rotonde, près du four. Il était alimenté en eau depuis l'ouest par une canalisation qui file au-dessus du mur sud du foyer, en partie haute.

Le système de chauffage du bain prend place au cœur même de l'édifice, comme il est de coutume dans les bains grecs d'Égypte. Le four est formé d'une bouche, ménagée entre deux maçonneries de pierre, dont une meule dormante placée sur le chant.

Les bains ont au moins connu deux phases de fonctionnement (le bâtiment date très certainement du III $\mathrm{s}$. av. J.-C., comme en attestent les monnaies trouvées dans la démolition, cf. infra), avant qu'ils ne soient abandonnés en tant que bains. Le plan du bâtiment reste sensiblement le même durant les deux phases, avec un agrandissement de ses annexes et un réaménagement qui concerne surtout l'angle sud-ouest des salles balnéaires.

\subsubsection{Le quartier nord-ouest du village}


Le quartier nord-ouest se caractérise par la présence d'un bâtiment aux larges dimensions, qui contraste avec l'organisation en constructions et pièces plus petites du reste du village.

\subsubsection{Bâtiment principal} zone et peut-être contemporain des bains. Il n'est desservi que par une seule entrée mais l'arasement de l'angle nord-ouest ne permet pas de la situer avec certitude. Il se développe vraisemblablement sur deux niveaux différents, suivant la déclivité naturelle de la terrasse alluviale sur laquelle se situe le village. Le matériel découvert dans les différentes pièces est pauvre, de sorte qu'il est difficile de donner une fonction à ce bâtiment, visiblement important dans le fonctionnement du village.

nord du bâtiment, qui occupe toute la partie nord de l'édifice, se divise en trois espaces : un petit podium, un espace central et une exèdre semi-circulaire. Des deux pièces au sud, la première se compose d'un petit aménagement contre le mur sud et d'un foyer dans le coin, la seconde est caractérisée principalement par un grand aménagement au sol fait de meules allantes. Les traces d'occupation dans le bâtiment sont extrêmement ténues et n'autorisent pas à en identifier la fonction.

\subsubsection{2. Îlot 344}

La fouille a mis au jour une série de 8 cellules et de 3 espaces extérieurs.

Fig. 3. Secteur nord-ouest du village de Ghozza en fin de fouille (T. Faucher).

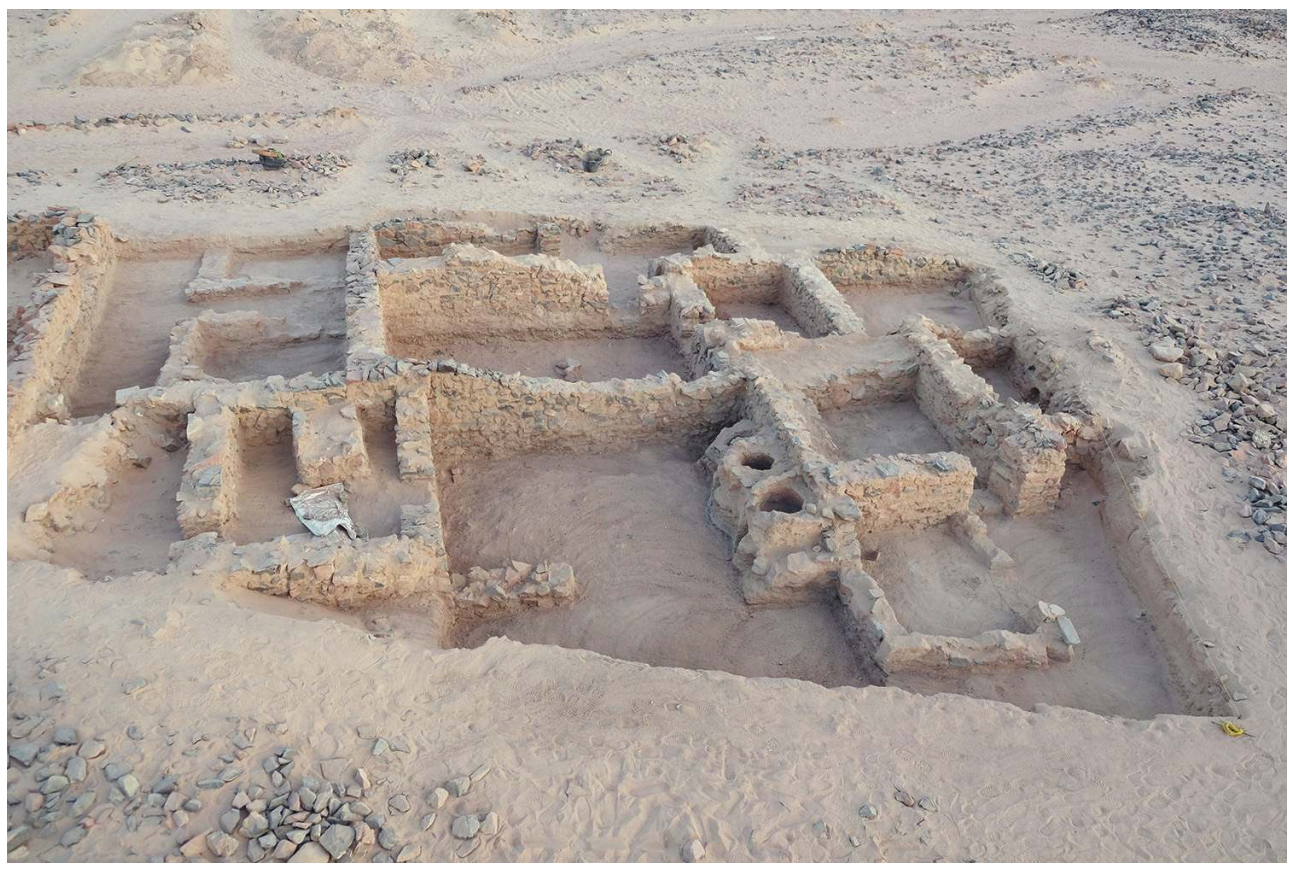

(C) MAFDO, 17152_2020_NDMPF_003

Il est encore trop tôt pour mettre en évidence une structuration de cet îlot mais il ne semble pas qu'il ait été construit à partir de modèles préétablis et uniformément dans le temps. Tout comme cela était visible dans le sondage du bâtiment 301, l'installation 
primitive du quartier s'est faite au sommet de la terrasse alluviale en s'accommodant du pendage naturel.

Si la majorité des cellules se sont construites au même moment, après l'édification du bâtiment 1 , il existe dans deux cellules, et dans l'espace le plus à l'ouest, des fosses, dans lesquelles du matériel céramique a été retrouvé. Ces fosses se retrouvent sous le niveau des premiers sols de circulation et n'étaient pas visibles avant un dégagement complet de ceux-ci.

Dans l'ensemble des cellules, un sol en mouna a été mis au jour, sans qu'aucun aménagement de type dallage ou autre soit mis en évidence. Cela ajoute au côté fruste de ces espaces pour lesquels aucun enduit ou même chaulage des murs n'a pu être enregistré. La première occupation de la zone est caractérisée par des niveaux de paille et de sable assez pauvres en matériel céramique. Les premiers aménagements sont peu nombreux. Il existe une sorte de réservoir en mouna dans la partie orientale d'une pièce, quelques foyers épars, mais c'est surtout les banquettes installées dans une cabane qui retiennent l'attention. Disposées sur trois côtés de la pièce, elles indiquent clairement une cellule de vie, au moins à fonction de dortoir. Cette pièce communique vers l'extérieur avec une seule porte, au sud, qui donne sur un petit enclos aménagé grâce à un modeste muret fait de pierres et de mouna.

Une grande phase de remaniement de la zone semble s'être effectuée en une seule étape. On installe dans deux espaces, vraisemblablement des zones ouvertes ou semiouvertes, deux séries de fours de type kanoun. L'installation de ces structures de combustion a dû s'accompagner de la mise en place de murets pour définir de nouveaux espaces.

La réoccupation de la zone à l'époque romaine semble avoir été extrêmement ténue. Ces espaces devaient déjà être largement recouverts par le sable et les bâtiments existants (les bains, le grand bâtiment) ont suffi aux habitants du fortin pour leurs activités annexes.

\subsubsection{Conclusions sur le village ptolémaïque}

Les découvertes faites dans la zone nord-ouest du village sont, à plusieurs titres, exceptionnelles. Il s'agit en effet du premier village ptolémaïque de mineurs fouillé dans le désert Oriental, dont les vestiges diffèrent, dans leur nature et leur organisation, de ceux mis au jour à Samut Nord, peut-être en raison d'une chronologie légèrement différente (le site de Samut Nord est occupé à la fin $\mathrm{du}_{\mathrm{IV}}^{\mathrm{e}} \mathrm{s}$., celui de Bir Samut au $\mathrm{III}^{\mathrm{e}}$ et $\mathrm{II}^{\mathrm{e}} \mathrm{s}$. av. J.-C.) et d'un contexte de production de nature distincte qu'il nous faudra éclaircir. Si on pouvait craindre que la zone fût extrêmement pauvre en matériel, ce n'est pas le cas. Plusieurs pièces de l'îlot ont donné un abondant matériel céramique issu de l'abandon du village, tout comme les différentes zones de dépotoirs renseignent sur la vie du village à ses différents stades d'occupation. La découverte conjointe d'un nombre important d'ostraca administratifs et comptables en grec et en démotique offre une fenêtre inédite sur le fonctionnement d'un tel village minier à cette époque. Il est d'ailleurs surprenant de trouver ce matériel en place. Nous avions bien constaté à Samut Nord que le retrait organisé des habitants et des troupes gérant les bâtiments n'avait laissé que très peu de matériel, chacun emportant avec soi ses biens respectifs. La situation est différente à Ghozza. Le matériel mis au jour est 
d'autant plus impressionnant que la zone fouillée ne représente pas plus de $10 \%$ du village.

\section{Deir el-Atrash}

Julie Marchand, Joachim Le Bomin blocs de grauwacke, il s'agence dans un carré irrégulier de $55 \mathrm{~m}$ autour d'une grande
fosse centrale à l'intérieur de laquelle se trouve un puits maçonné. Une seule entrée
flanquée de tours carrées, préservées sur près de $5 \mathrm{~m}$ de hauteur, ouvre le fort dans son
flanc sud. Des tours s'élèvent également à chaque angle de l'édifice. Une citerne
quadrangulaire est installée dans l'angle sud-ouest du fort et semble connectée au puits
ainsi qu'à une ligne d'abreuvoirs situés à l'extérieur à l'ouest aboutissant à un bassin de
récupération d'eau. L'originalité des structures visibles tient à l'utilisation importante
de la brique crue, employée de manière systématique, en dehors des courtines, sur
l'ensemble des parties hautes des élévations, pour les couvertures des espaces internes
et pour les tours d'entrée. Enfin, à l'est, le fort est adjoint par un ensemble affleurant la
surface présentant une succession de couloirs d'orientation nord-sud délimités dans un
périmètre de $56 \times 42 \mathrm{~m}$.

\subsection{L'entrée}

Le système d'entrée du fort a fait l'objet de l'attention principale de cette première campagne. L'excellent état de conservation avec des tours de $5 \mathrm{~m}$ de hauteur laissait envisager une étude précise du système et de son évolution. La présence d'un monticule de sable et de matériaux d'architecture dans le passage d'entrée suggérait la préservation des niveaux de circulation. 
Fig. 4. Entrée du fort de Deir el-Atrash (J. Le Bomin).

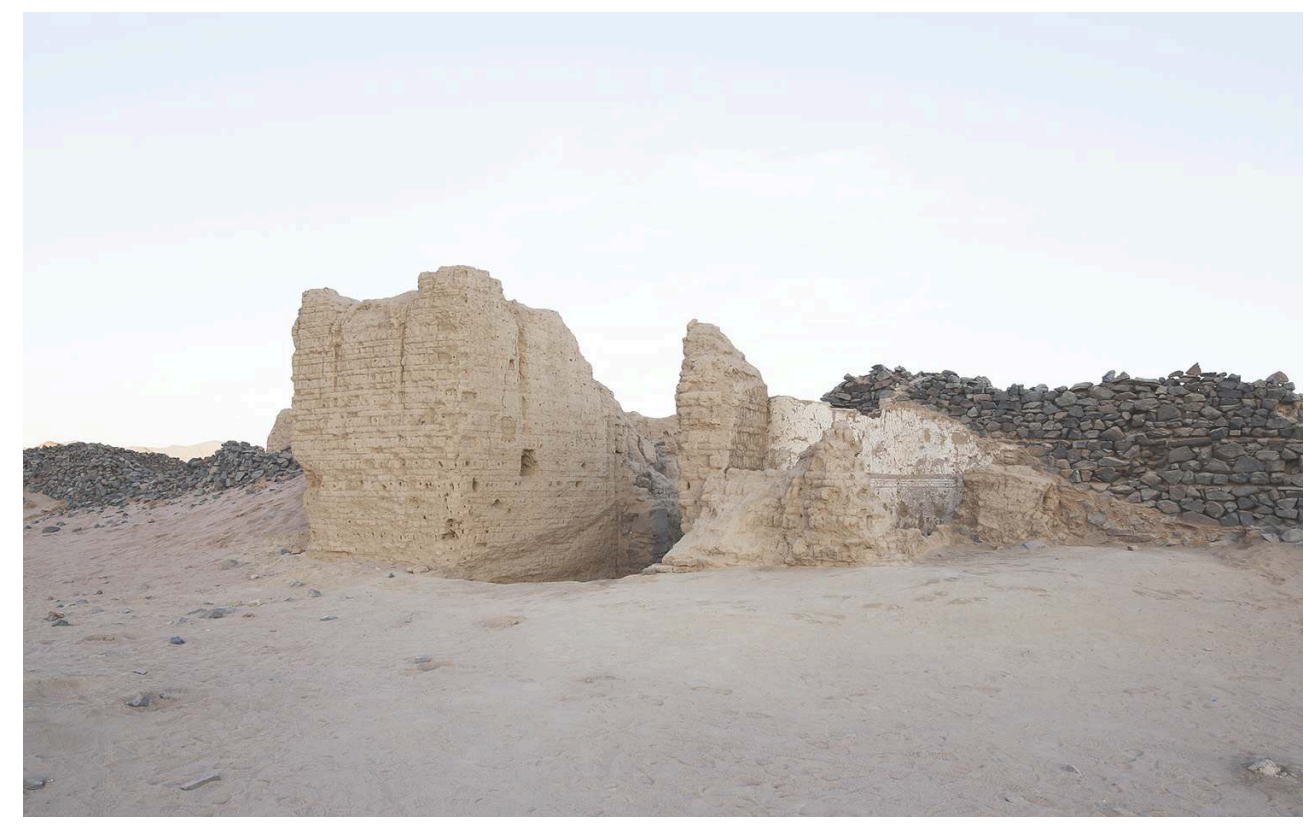

(c) MAFDO, 17152_2020_NDMPF_004

Un sondage a été pratiqué sur toute la surface du passage d'entrée sans atteindre le plancher naturel. Le nettoyage de la tour extérieure est a permis d'identifier la première phase d'aménagement du fort avec la découverte d'une tour semi-circulaire chaînée à la courtine. Seule la moitié orientale de la tour subsiste, l'autre moitié ayant été spoliée lors de la construction de la tour carrée plus tardive. Les fouilles réalisées dans le passage ont permis de mettre au jour les jambages de la porte d'entrée ainsi que les ancrages du linteau de porte aujourd'hui disparu.

31 À l'heure actuelle, les niveaux d'occupation liés à cette phase restent relativement ténus. Avec le sondage réalisé dans le dépotoir on peut, avec toutes les précautions qui s'imposent, proposer une datation pour la construction du fort et sa première phase d'occupation, à la période antonine, sous l'empereur Hadrien au plus tard.

Les éléments dont nous disposons pour l'instant laissent penser qu'une période d'inoccupation intervient assez rapidement après la phase précédente et dure jusqu'à la fin $d u \mathrm{IV}^{\mathrm{e}}$ siècle. Cette deuxième phase est marquée par le réaménagement massif du système d'entrée qui est intégré au sein des nouvelles constructions qui se matérialisent principalement par l'adjonction de quatre tours quadrangulaires en briques crues. Les niveaux d'occupations les plus récents sont rassemblés dans le passage d'entrée. Le matériel y est également peu abondant et daté de la fin du IV $\mathrm{V}^{\mathrm{e}}$ début du ve siècle.

\subsection{Le dépotoir}

Le dépotoir est situé à l'extérieur, au sud du fort, face à l'entrée et forme un monticule arasé à peine à $50 \mathrm{~cm}$ de la surface de la terrasse sur laquelle est installé le fortin. Les niveaux inférieurs offrent un matériel très fragmenté et un peu moins abondant que pour le niveau supérieur rassemblant de la céramique datée du début $d u{ }{ }^{e} s$., du 
textile, du bois, de la faune et du cuir. Les niveaux supérieurs du dépotoir se distinguent essentiellement par le mobilier daté de la fin du Ive-début du ve siècle.

\subsection{L'aile ouest}

À l'intérieur du fort, les investigations se sont principalement concentrées au niveau de l'aile est dans laquelle un nettoyage approfondi a été réalisé sur une surface d'environ $70 \mathrm{~m}^{2}$ sur toute la largeur de l'aile.

Fig. 5. Aile ouest du fort de Deir el-Atrash (G. Pollin).

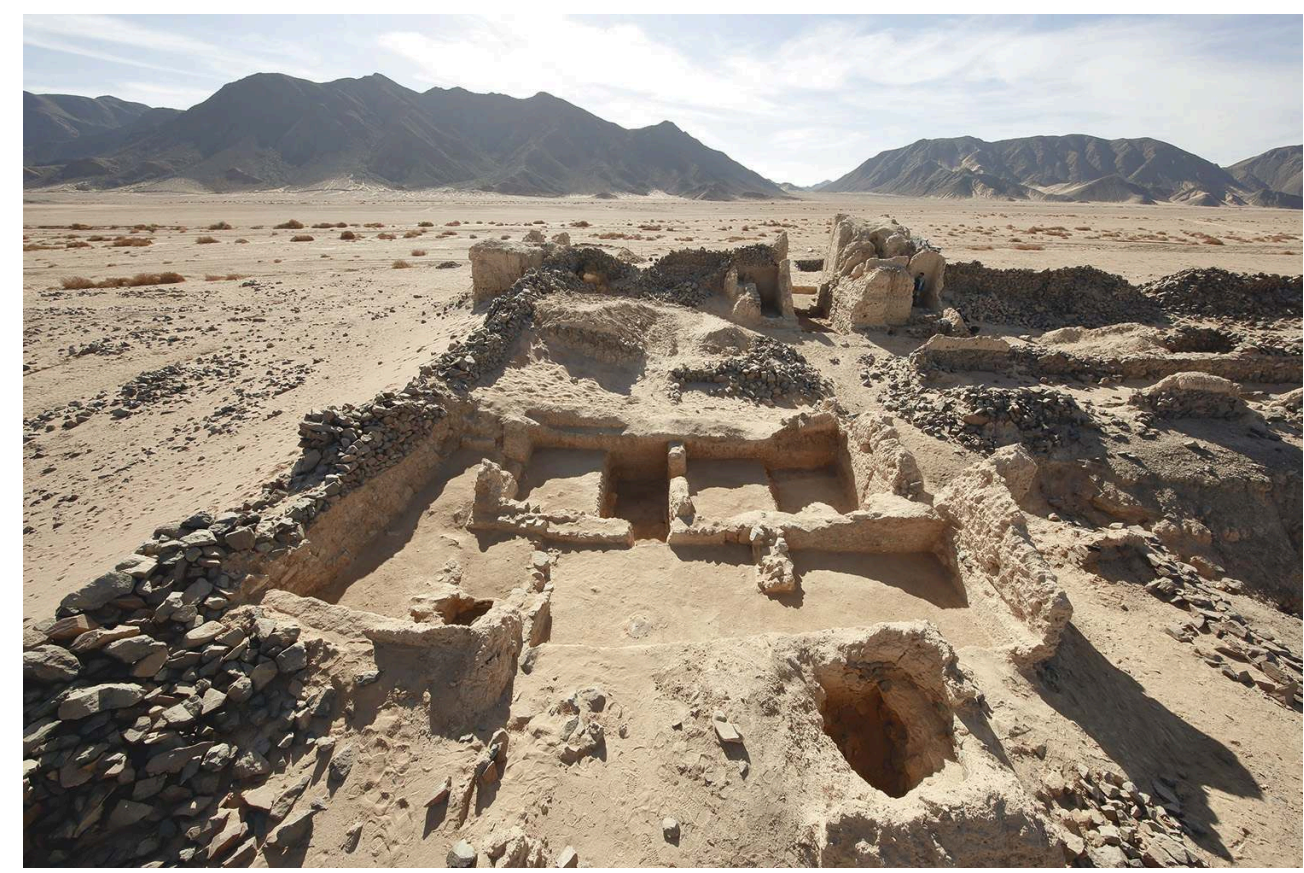

(C) MAFDO, 17152_2020_NDMPF_005

Dans cet espace, les élévations en briques crues bien conservées jusqu'au départ des voûtes offrent la possibilité d'étudier la structuration des pièces. Des indices de réaménagements semblent confirmer les observations réalisées dans les autres secteurs de fouille même si la chronologie reste ici à préciser. Pour l'instant, les niveaux de nettoyage et de comblement des pièces relatifs à l'abandon offrent du matériel de la fin $\mathrm{du} \mathrm{IV}^{\mathrm{e}}$ et du $\mathrm{v}^{\mathrm{e}}$ siècles.

\subsection{L'espace central et le puits}

Une tranchée d'orientation est-ouest a été implantée contre la margelle du puits. Il est peu aisé de définir clairement la relation de ce niveau, anthropique au regard du matériel collecté, avec la margelle du puits à savoir s'il s'agit d'un dépôt antérieur ou non à son creusement. Au-dessus, s'étend un monticule à pendage subhorizontal sans aucun mobilier qui peut être interprété comme une couche de curage du puits. 


\subsection{Les « animal lines »}

Le dernier secteur à avoir fait l'objet d'investigation lors de cette campagne est situé à l'extérieur du fort à l'est. La présence d'une grande quantité de cendres, sans pour autant que les structures adjacentes présentent des traces de rubéfaction, reste inexpliquée. Force est de constater que l'hypothèse de structures réservées à l'accueil des animaux reste la plus vraisemblable en l'état des investigations.

\subsection{La peinture de la tour d'entrée}

La fouille du système d'entrée, qui avait pour but d'étudier les différentes phases de construction du complexe, a permis la découverte d'une vaste peinture sur une tour initiale. Le décor est polychrome, sur un fond de mouna, couvert d'un badigeon. La frise mesure donc plus de $2 \mathrm{~m}$ de long et mesure $1,80 \mathrm{~m}$ de hauteur.

Tout d'abord, un cavalier est représenté sur un cheval en mouvement, au sommet de la tour ronde, sur une bande rouge épaisse, à hauteur d'yeux. Au-dessus de lui, s'allonge un serpent vert sombre, gueule ouverte. L'autre importante scène du programme est située sur la courtine : il s'agit d'une caravane de quatre chameaux, avançant à la file indienne, et menés par un homme vêtu d'une tunique à clavi bruns tenant a priori une longe de sa main droite. Le registre inférieur consiste principalement en motifs de rinceaux de vigne enroulés sur eux-mêmes. Ils se développent sur la courtine et à l'angle de la tour.

\subsection{Conclusion}

Après cette première campagne de fouille sur le praesidium de Deir el-Atrash, il est possible d'apporter de nombreux éléments nouveaux concernant la mise en place du complexe et sa chronologie. Des indices solides permettent d'envisager une fondation durant la période antonine sous les empereurs Trajan ou Hadrien suivi d'une occupation relativement courte seulement reprise trois siècles plus tard, à la fin du IV ou au début $d u v^{e} s$. qu'il faut peut-être associer à la reprise des activités des carrières du Porphyritès à l'époque byzantine. L'état de conservation du fortin offre des perspectives remarquables pour l'étude de l'architecture des édifices militaires du désert tant à la période romaine qu'à l'époque byzantine. La découverte exceptionnelle et inédite d'une fresque peinte, le développement du dispositif d'entrée, les pièces voûtées à la nubienne sont des exemples rares, preuves de la nécessité d'en poursuivre l'examen minutieux.

\section{3. Études géographiques, topographiques et géologiques}

\subsection{Géographie et environnement de la région de Ghozza et el- Atrash}


41 Dans le cadre de l'étude des sites de Ghozza et du Deir el-Atrash situés dans un milieu à l'aridité prononcée, l'analyse de la géographie et de l'environnement à plusieurs échelles, de la région au site, est essentielle. Actuellement, la zone d'étude recevrait moins de $5 \mathrm{~mm}$ de précipitations annuelles moyennes alors que l'évaporation potentielle dépasse $4000 \mathrm{~mm}$ par an. Ce contexte d'hyperaridité met donc en question les ressources disponibles pour les occupants et les exploitants du désert, ainsi que les contraintes et les risques liés aux systèmes morphogénétiques des milieux arides.

Ghozza et el-Atrash se situent dans la partie ouest des collines de la mer Rouge et appartiennent au bassin versant du ouadi Qena (qui se raccorde au Nil). Ils se situent donc en dehors du bassin concerné par le Système aquifère des grès nubiens, et ne peuvent pas bénéficier directement de sa fourniture en eau, particulièrement importante.

Les sédiments du ouadi el-Atrash témoignent d'écoulements très peu compétents lors des crues, ce qui explique l'omniprésence du sable, la formation de micro-dépressions emplies de limons et d'argiles, et le nombre très réduit d'éléments supérieurs aux sables grossiers. Les crues ne semblent donc pas pouvoir constituer une contrainte majeure pour l'occupation du site. Dans le ouadi el-Ghozza, au contraire, on observe des indications de crues plus puissantes, capables de déplacer des galets et quelques petits blocs, les pentes étant plus fortes et les chenaux d'écoulement plus étroits et plus incisés.

L'ensemble de la région se caractérise par un contraste fort entre les fonds de ouadis majoritairement sableux ou sablo-limoneux, où la végétation est concentrée, et des versants et des crêtes rocheuses ou couverts de colluviaux totalement dénués de plantes. Sur les versants, même les secteurs comportant des sédiments fins étaient dénués de végétation en raison du manque d'eau.

\section{2. Étude topographique}

Damien Laisney, Alexandre Rabot

\subsubsection{Ghozza}

Située du côté de la berge droite concave du large méandre que décrit le cours du ouadi el-Ghozza qui vient buter sur un éperon massif haut d'une quarantaine de mètres, la zone du fort se trouve sur un lambeau de cône d'affluents venant du nord. Cette position hydrogéologique particulière permet en ces lieux de bénéficier d'une source d'eau accessible provenant des écoulements hydrauliques du cône et/ou du ouadi. La terrasse alluviale sur laquelle est construit le village se situe quasiment au milieu du ouadi el-Ghozza. Elle est globalement orientée NE/SW et sa forme a été façonnée par les aléas du cours d'eau. Vis-à-vis des lits mineurs qui la cernent, son élévation est autour du mètre et, dans le paysage, sa silhouette passerait sans doute plus inaperçue sans la densité des constructions établies à cet endroit. D'ailleurs, comme pour le lambeau de cône sur laquelle se trouve la zone du fort, ces installations ont " armé » la terrasse contre l'érosion et il est vraisemblable que depuis l'Antiquité le village ait finalement subi peu de destructions majeures dues aux crues. Mis à part un bâtiment isolé sis à l'extrémité sud-ouest de la terrasse, le village occupe la totalité de la terrasse et couvre 
une surface totale de $5250 \mathrm{~m}^{2}$. Il s'agit d'un habitat groupé, agencé selon un plan plus ou moins orthogonal et régulier.

Quelque $200 \mathrm{~m}$ au sud du village, un creusement a été aménagé sur la rive gauche du ouadi el-Ghozza. En réalité, cet aménagement hydraulique a été implanté à cheval sur la berge et le lit du cours d'eau : cette position lui permet de bénéficier des écoulements provenant du ouadi et/ou des affluents des cônes situés au sud.

\subsubsection{Deir el-Atrash}

Le site archéologique est implanté au milieu du ouadi al-Atrash à une altitude située autour de $385 \mathrm{~m}$ dans une vallée très ensablée et très plane: sa largeur dépasse les $600 \mathrm{~m}$ et sa pente moyenne est de $0,5 \%$. Plusieurs tombes subcirculaires, généralement pillées avec à proximité des tessons de céramique d'époque romaine ont été repérées aux alentours du fort. Mises à part ces sépultures, les vestiges archéologiques sont essentiellement concentrés sur une terrasse alluviale profilée par les écoulements du cours d'eau. Nous noterons ici à quel point les constructions installées sur cette terrasse de ouadi ont pu l'« armer » et limiter ainsi son érosion. En dehors du pourtour de la forteresse où le sable éolien s'est fortement accumulé contre les murs d'enceinte, l'altitude actuelle de la terrasse est entre $50 \mathrm{~cm}$ et $1 \mathrm{~m}$ plus haut que les lits mineurs du ouadi qui l'entourent.

\section{3. Étude géologique et minière}

Markos Vaxevanopoulos

Les recherches ont été menées sur le district minier entourant le site de Ghozza, à la fois au nord-ouest et au sud. Les prospections n'ont couvert que le tiers de la zone minière et les résultats sont donc préliminaires. Une vingtaine d'entrées de mines ont été localisées; deux réseaux miniers ont été cartographiés alors qu'une galerie a fait l'objet d'une fouille. 
Fig. 6. Galerie de mine dans le sud du district minier de Ghozza (M. Vaxevanopoulos).

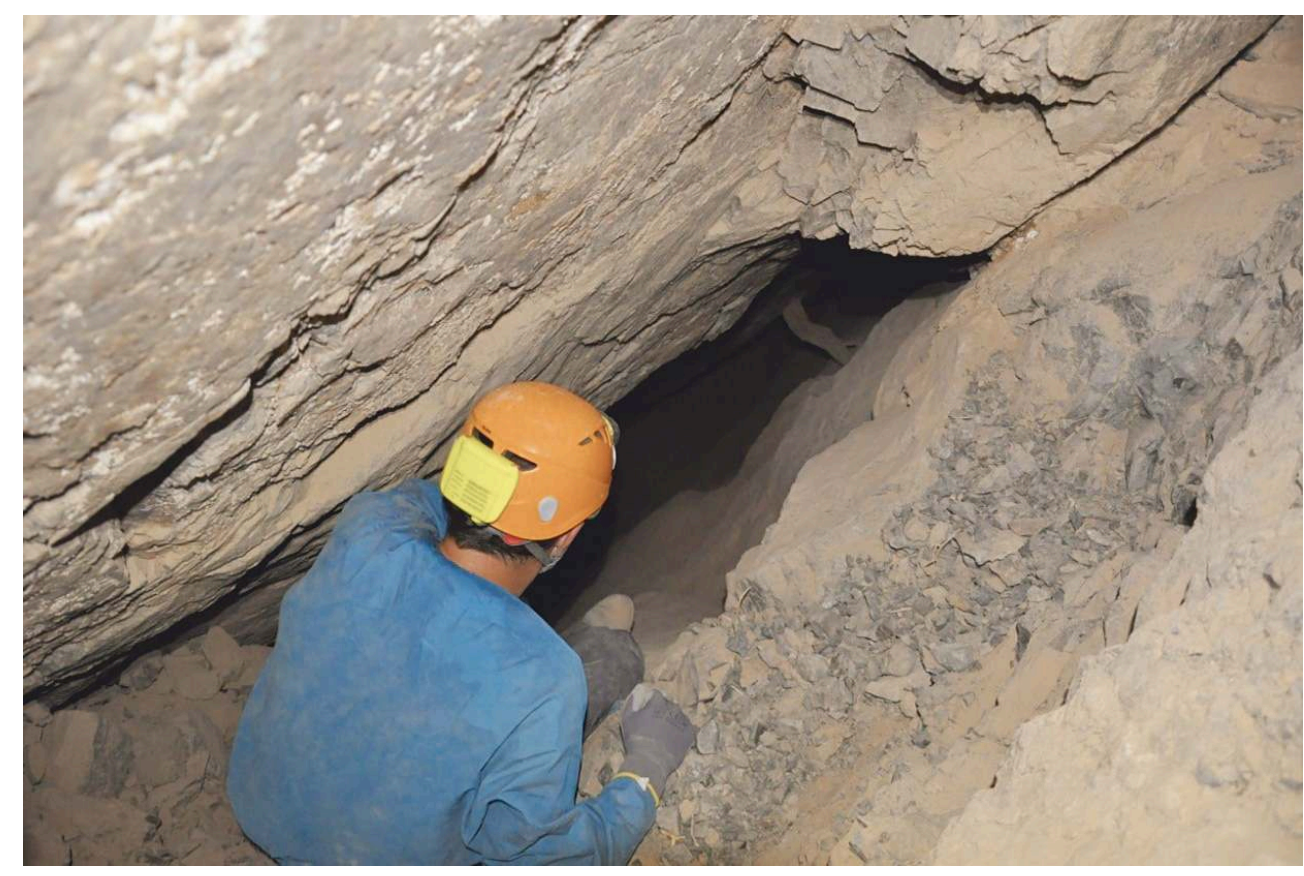

(c) MAFDO, 17152_2020_NDMPF_006

$49 \mathrm{Au}$ nord, les recherches se sont focalisées sur plusieurs veines de quartz aurifère d'orientation nord-est/sud-ouest, incluses dans différentes couches de greywacke, de siltite, des conglomérats, et des bandes d'argile métamorphiques et pliées. Les galeries se situent dans la partie latérale de ce pli. Il a généré une circulation hydrothermale et a amené au dépôt du quartz. La plus longue galerie s'étend sur $55 \mathrm{~m}$, sur une hauteur de $11 \mathrm{~m}$. On peut observer, ci et là, les traces de l'extraction sous la forme de coups de pics.

$50 \mathrm{Au}$ sud, un réseau de galeries s'étend sur plus de $70 \mathrm{~m}$ à une profondeur maximale de $18 \mathrm{~m}$, à une inclinaison de $60 / 70^{\circ}$. Au nord de la plus grande chambre, furent découverts deux paniers remplis de minerais mais coincés sous une importante quantité de stériles.

51 En complément de cette étude, nous avons enregistré 482 meules à broyer le minerai réparties sur le site de Ghozza et collecté 28 échantillons de roche pour une étude microscopique.

\section{4. Étude de matériel}

\subsection{Céramique}

\subsubsection{Ghozza}

Jennifer Gates-Foster

52 La céramique de Ghozza provient essentiellement de trois zones du site: le fortin romain, le complexe balnéaire et les habitations adjacentes. Le matériel céramique est abondant dans toutes les zones étudiées et apportent des éléments d'étude pour une large variété d'activités domestiques prenant place dans ces espaces, particulièrement en ce qui concerne le stockage, la préparation et la consommation de la nourriture. Le matériel retrouvé sur le site suggère trois phases d'occupation principales dans le $\mathrm{III}^{\mathrm{e}} \mathrm{s}$. 
av. J.-C., dans le $\mathrm{II}^{\mathrm{e}}$ s. av. J.-C., ainsi qu'au cours de la deuxième moitié du $\mathrm{I}^{\mathrm{er}}$ s. apr. J.-C. et au tout début du $\mathrm{II}^{\mathrm{e}}$ s. apr. J.-C.

\subsubsection{Deir el-Atrash}

J. Marchand

53 Le matériel céramique de Deir el-Atrash conduit à dater la dernière occupation du fortin de la période romaine tardive, plus précisément du v s. apr. J.-C., en raison de la présence d'amphores LR7 (produites en Moyenne Égypte), ainsi que de tardives AE3 (à pâte alluviale). Les assemblages se composent également de quelques importations : LR1, 3, 4 et 5/6. Le groupe égyptien se compose de cruches à pâte alluviale et de marmites. Très peu de vaisselle de table fut enregistrée. Il est à noter la présence de pots à pigeons ainsi que celle probable de pots à saqqia.

54 A l'extérieur du fort, dans le dépotoir, les derniers niveaux se composent également de matériel $\mathrm{du}_{\mathrm{V}}^{\mathrm{e}} \mathrm{s}$. apr. J.-C. Sous ces couches, le faciès du matériel change complètement et apparaît alors des marqueurs d'une présence romaine datant du $\mathrm{II}^{\mathrm{e}} \mathrm{s}$. apr. J.-C. Les assemblages d'amphores égyptiennes 3, de marmites, d'Eastern Siggilata A et de céramique fine égyptienne sont très proches de ceux observés dans les carrières du Mons Claudianus et du Porphyritès.

\subsection{Ostraca}

Hélène Cuvigny, Adam Bülow-Jacobsen

\subsubsection{Ghozza}

\subsubsection{Ostraca du praesidium}

Sur les 135 ostraca enregistrés, 46 ont été trouvés à l'intérieur du praesidium, dont ils ont révélé le nom : Berkou (Brpkov). Ce toponyme est vraisemblablement égyptien (ce

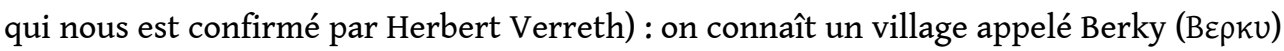
dans le nome Oxyrhynchite. Comme on peut supposer que ce nom indigène était déjà celui du village ptolémaïque, les ostraca de Ghozza seront appelés O.Berkou.

Les O.Berkou romains sont tous en grec, à l'exception d'un latin trop effacé pour être exploitable. Un ostracon est daté de l'an 10 de Domitien (AD 90), un autre d'un an 11, sans doute aussi de Domitien, mais le nom de l'empereur est perdu (inv. 54 et 70). Malgré la minceur de ce corpus, des liens ont pu être établis avec les ostraca d'Umm Balad (O.KaLa.).

La plupart des O.Berkou sont des lettres échangées avec les stations immédiatement voisines. La plus remarquable est O.Berkou inv. 39, écrite par un chasseur qui dit que ce n'est pas la saison des onagres, mais qu'en revanche il a capturé deux gazelles.

\subsubsection{Ostraca grecs du village ptolémaïque}

88 ostraca provenant du village ont été enregistrés (54 grecs and 33 démotiques et bilingues). Une lettre en grec a en outre été trouvée dans une galerie de mine (inv. 40).

Dans le village, comme c'était le cas à Bi'r Samût, les lettres en grec, au nombre de 3, sont rares. Le corpus grec est essentiellement constitué de comptes relatifs au travail 
de la main-d'œuvre. Le plus remarquable de ces comptes (inv.18) est inscrit sur la panse d'une jatte que J.Gates-Foster date du début du II $^{\mathrm{e}} \mathrm{s}$. av. J.-C. Beaucoup de comptes enregistrent le rendement quotidien des broyeurs de minerai, la farine obtenue étant mesurée en artabes et matia $(=1 / 12$ d'artabe). Certaines des personnes mentionnées dans les comptes de la même série sont des femmes. On reconnaît aussi parmi cette main-d'œuvre plusieurs noms juifs. Un compte de nourriture mentionne d'ailleurs le sabbat (inv. 116). Les scribes hellénistes qui contrôlaient jour après jour le rendement des broyeurs n'étaient pas dépourvus de culture grecque: deux ostraca littéraires ont été mis au jour dans le village, dont l'un, opisthographe, est un hymne à Dionysos.

\subsubsection{Deir el-Atrash}

60 Sur les 42 ostraca inventoriés, aucun ne comporte le nom antique du site. Les seuls toponymes mentionnés sont Berkou et le Porphyrites. 22 ostraca issus des couches anciennes du dépotoir datent $\mathrm{du} \mathrm{I}^{\mathrm{er}}$ et $\mathrm{du} \mathrm{II}^{\mathrm{e}}$ s. apr. J.-C. ; 6 d'entre eux sont en latin. Les vingt autres ostraca datent $d u \mathrm{IV}^{\mathrm{e}}$ ou peut-être plutôt du début du ve $\mathrm{s}$. apr. J.-C. et sont tous en grec.

61 L'US $10.09\left(\mathrm{IV}^{\mathrm{e}}-\mathrm{V}^{\mathrm{e}} \mathrm{s}\right.$.) a livré 7 bons d'orge (mesurée en modii et sextarii) à distribuer à des ânes originaires de plusieurs nomes de Moyenne Égypte et du Delta. Ces animaux pourraient avoir été réquisitionnés au titre de la liturgie appelée onelasia pour travailler dans le désert, ou simplement ravitailler les sites qui fonctionnaient à cette époque : peut-être plus le Porphyrites, où l'exploitation est indirectement documentée pour la dernière fois en 335 (P.Horak 12), mais sans doute Abû Sha'r, où un fort avait été construit sur la mer Rouge vers 310 pour assurer la protection du commerce maritime. Abandonné par l'armée à la fin du IV ${ }^{\mathrm{e}}$ s. apr. J.-C., le site avait accueilli une communauté monastique.

\subsection{Petits objets}

Mariola Hepa, Noémi Villars

$62 \mathrm{Au}$ cours de la saison 2020, 201 "petits objets » furent enregistrés, dessinés et photographiés par la mission. Ces découvertes comprennent entre autres 56 fragments de faïence (vaisselle et perles), 16 fragments de vaisselle en verre, 35 fragments en terre cuite (lampes ou culs d'amphores travaillés). D'autres objets furent enregistrés, comme plus de 60 fragments en matériau lithique (outils et vaisselle), 5 fragments de métal (notamment un couteau intact), un bol en bois, des coquillages, ainsi que des objets en bronze et en plomb.

\subsection{Les monnaies}

T. Faucher

Cette année, 6 monnaies en alliage cuivreux furent découvertes lors des fouilles. À Ghozza, une monnaie provient du fortin romain; elle a été frappée en l'an 10 de Vespasien. Quatre monnaies proviennent de la zone des bains, dans le village, et ont toutes été frappées dans la deuxième moitié du $\mathrm{III}^{\mathrm{e}} \mathrm{s}$. av. J.-C. Elles appartiennent toutes à la série 3 des monnaies de bronze lagides (frappées de 261 à 240). 

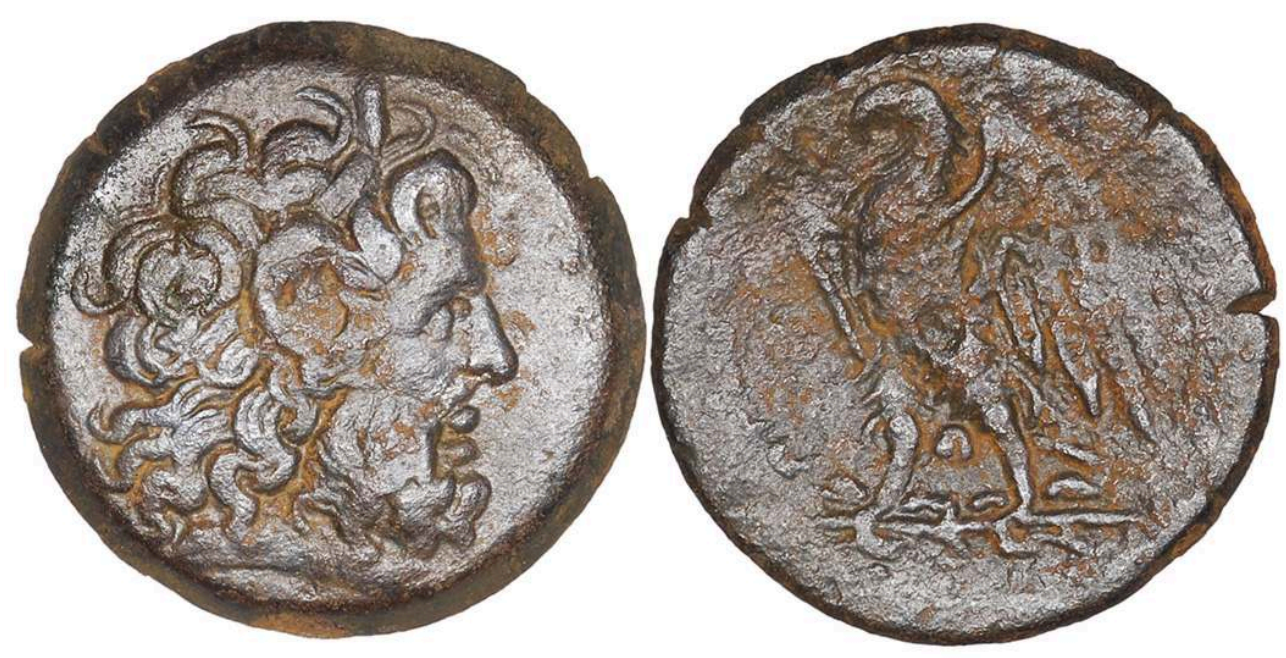

0

1

$2 \mathrm{~cm}$

Find_Gho_040

(c) MAFDO, 17152_2020_NDMPM_001

Toutes ces monnaies sont en très bon état de conservation et ont vraisemblablement été perdues à une date assez proche de leur moment d'émission. À Deir el-Atrash, une monnaie en très mauvais état a été découverte, qu'on ne peut malheureusement pas mieux dater que des $\mathrm{IV}^{\mathrm{e}}$ et $\mathrm{V}^{\mathrm{e}}$ siècles.

\section{Publications, valorisation, travaux universitaires}

\subsection{Monographies}

- Bérangère Redon, Thomas Faucher (éd.), Samut Nord. L'exploitation de l'or du désert Oriental à l'époque ptolémaïque, FIFAO 83, Le Caire, Ifao, 2020.

- Damien Agut-Labordère, Bérangère Redon (éd.), Les vaisseaux du désert et des steppes. Les camélidés dans l'Antiquité (Camelus dromedarius et Camelus bactrianus), Archéologie(s) 2, Lyon, MOM édition, 2020, livre en ligne sur OpenEdition Books, https:// books.openedition.org/momeditions/8457, consulté le 7 avril 2021.

- Hélène Cuvigny (éd.), Blemmyes: New Documents and New Perspectives, DFIFAO, Le Caire, Ifao, à paraître.

\subsection{Articles parus ou à paraître}

- Maire-Pierre Chaufray, "Les chameaux dans les ostraca démotiques de Bi'r Samut ", in Damien Agut-Labordère, Bérangère Redon (éd.), Les vaisseaux du désert et des steppes. Les camélidés dans l'Antiquité (Camelus dromedarius et Camelus bactrianus), Archéologie(s) 2, 
Lyon, MOM édition, 2020, article en ligne sur OpenEdition Books, https:// books.openedition.org/momeditions/8567, consulté le 7 avril 2021.

- Hélène Cuvigny, L'élevage des chameaux sur la route d'Edfou à Bérénice d'après une lettre trouvée à Bi'r Samut (III siècle av.J.-C.), in Damien Agut-Labordère, Bérangère Redon (éd.), Les vaisseaux $d u$ désert et des steppes. Les camélidés dans l'Antiquité (Camelus dromedarius et Camelus bactrianus), Archéologie(s) 2, Lyon, MOM édition, 2020, article en ligne sur OpenEdition Books, https://books.openedition.org/momeditions/8572, consulté le 7 avril 2021.

- Martine Leguilloux, «Camelus ou Equus? Le rôle des dromadaires dans les stathmoi et praesidia du désert Oriental d'Égypte ", in Damien Agut-Labordère, Bérangère Redon (éd.), Les vaisseaux du désert et des steppes. Les camélidés dans l'Antiquité (Camelus dromedarius et Camelus bactrianus), Archéologie(s) 2, Lyon, MOM édition, 2020, article en ligne sur OpenEdition Books, http://books.openedition.org/momeditions/8577, consulté le 7 avril 2021.

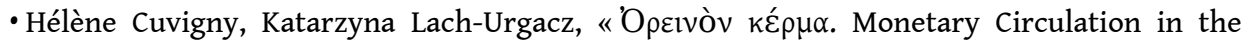
Praesidia of the Eastern Desert during the Principate », in Thomas Faucher (éd.), Money Rules! The Monetary Economy of Egypt, from Persians until the Beginning of Islam, BiEtud 176, Le Caire, Ifao, 2020, p. 309-339.

- Maël Crépy, Louis Manière, Bérangère Redon, « Roads in the Sand: Using Data from Modern Travelers to Reconstruct the Ancient Road Networks of Egypt Eastern Desert », in Tuna Kulayci (éd.), The Archaeologies of Roads, University of Florence, article soumis en février 2020.

- Jennifer Gates-Foster, Bérangère Redon, Melanie Godsey, «Domestic Activities in Alternative Settings: The Ptolemaic Fort at B'ir Samut, Egypt», in Caitlín Barrett, Jennifer Carrington (éd.), Households of Ptolemaic and Roman Egypt, Ithaca, Cornell University Press, accepted, expected 2021.

- Jennifer Gates-Foster, «Third Century BCE Supply Networks and Ptolemaic Transport Amphoras from 'Abbad and Bir Samut in Egypt's Eastern Desert », in Bérangère Redon (éd.), Networked Spaces: The Spatiality of Networks in the Red Sea and the Western Indian Ocean, Lyon, Maison de l'Orient et de la Méditerranée, submitted 2020.

- Jennifer Gates-Foster, «New Archaeological Evidence for the Indigenous Peoples of the Eastern Desert in the Ptolemaic and Roman Eras ", in Hélène Cuvigny (éd.), Blemmyes: New Documents and New Perspectives, DFIFAO, Le Caire, Ifao, à paraître.

- Bérangère Redon, Thomas Faucher, «Recent Discoveries of BE Arrowheads and Joppa Coins in the Eastern Desert of Egypt: In the Footsteps of Soldiers of the Ptolemaic Army ", Bulletin of the American Society of Oriental Research, à paraitre.

\section{BIBLIOGRAPHIE}

FOURNET, REDON 2017

Thibaud Fournet, Bérangère Redon, « Bathing in the Shadows of the Pyramids: Greek Baths in Egypt, Back to an Original Bath Model », in Bérangère Redon (éd.), Collective Baths in Egypt 2: New Discoveries and Perspectives, EtUrb 10, Le Caire, Ifao, 2017, p. 99-137. 
NOTES

1. FOURNET, REDON 2017, part. p. 114-117 et 119-120.

INDEX

Thèmes : IFAO

Année de l'opération : 2020

\section{AUTEURS}

THOMAS FAUCHER

Archéologue, numismate, IRAMAT-CRP2A, Bordeaux

BÉRANGÈRE REDON

Archéologue, CNRS-HiSoMA, Lyon

ADAM BÜLOW-JACOBSEN

Papyrologue, photographe

MAËL CREPY

Géographe, UMR 5189 HiSoMA, UMR 5133 Archéorient

HÉLÈNE CUVIGNY

Papyrologue, CNRS-IRHT, Paris

JENNIFER GATES-FOSTER

Céramologue, University of North Carolina at Chapel Hill

ISABELLE GONCALVES

Archéologue, doctorante, université de Lyon

\section{MARIOLA HEPA}

Archéologue, Swiss Institute for Architectural and Archaeological Research on Ancient Egypt

DAMIEN LAISNEY

Topographe, FR 3747 Maison de l'Orient et de la Méditerranée

JOACHIM LE BOMIN

Archéologue, UMR 5189 HiSoMA 
JULIE MARCHAND

Céramologue, UMR 5189 HiSoMA

\section{ALEXANDRE RABOT}

Spécialiste du SIG, HiSoMA, université Lumière Lyon 2

MARKOS VAXEVANOPOULOS

Géologue, ENS Lyon

NOÉMI VILLARS

Data Manager, CNRS-HiSoMA 